\title{
Decreased expression of survivin 2B in human pituitary adenomas. A preliminary study
}

\author{
Joanna Waligorska-Stachura', Nadia Sawicka-Gutaj ${ }^{1}$, Maciej Zabel ${ }^{2}$, \\ Wlodzimierz Liebert ${ }^{3}$, Pawel Gut ${ }^{1}$, Agata Czarnywojtek ${ }^{1,4}$, Marek Ruchala ${ }^{1}$
}

${ }^{1}$ Department of Endocrinology, Metabolism and Internal Medicine, Poznan University of Medical Sciences, Poznan, Poland

${ }^{2}$ Department of Histology and Embryology, Poznan University of Medical Sciences, Poznan, Poland

${ }^{3}$ Department of Neurosurgery and Neurotraumatology, Poznan University of Medical Sciences, Poznan, Poland

${ }^{4}$ Department of Pharmacology, Poznan University of Medical Sciences, Poznan, Poland

\begin{abstract}
Introduction. We aimed to investigate survivin and its splice variants DEx3 and $2 \mathrm{~B}$ expressions in pituitary adenomas and normal pituitary glands using immunohistochemistry.

Material and methods. The study group consisted of eight pituitary adenomas: five of non-functional tumors, two of GH-secreting tumors, and one PRL-secreting tumor. Eight healthy pituitary tissue samples obtained after autopsy served as controls.

Results. Survivin expression was found in $87.5 \%$ of the study group and $100 \%$ of the controls. A positive staining of survivin $2 \mathrm{~B}$ was found in $62.5 \%$ of pituitary adenomas and $100 \%$ of controls. Survivin DEx3 was recognized in $25 \%$ of pituitary adenomas and $12.5 \%$ of normal pituitary glands. There was significantly lower immunoreactivity of survivin $2 \mathrm{~B}$ in pituitary adenomas when compared with normal pituitary glands $(\mathrm{p}=0.0498)$.

Conclusions. Survivin and its splice variants might be involved to some extent in benign tumor growth of pituitary adenomas. However, survivin cannot be regarded as a candidate for targeted therapy or molecular biomarker of pituitary adenomas. (Folia Histochemica et Cytobiologica 2017, Vol. 55, No. 1, 21-25)
\end{abstract}

Key words: pituitary adenoma; surviving; survivin DEx3; survivin 2B; IHC

\section{Introduction}

Sporadic pituitary adenomas are frequently visualized during radiological examination or autopsy indicating their significant prevalence in up to $17 \%$ of the population [1]. Clinically relevant pituitary adenomas are less frequent, though they are among the most common tumors of the central nervous system [2, 3]. The pathogenesis of pituitary tumorigenesis still

Correspondence address: N. Sawicka-Gutaj, M.D., Ph.D.

Department of Endocrinology, Metabolism

and Internal Medicine

Poznan University of Medical Sciences

Przybyszewskiego 49 St., 60-355 Poznan, Poland

tel.: +486186913 30, fax: +48618691682

e-mail:nyha@o2.pl remains unclear [4-6]. Pituitary adenomas arise from monoclonal growth and pituitary tumor transforming gene (PTTG) plays an important role in their development $[7,8]$. However, the pathogenesis of pituitary adenomas is complex and many other factors are suggested to be involved in their development and progression [9-12].

Survivin is a member of the IAP (inhibitor of apoptosis protein) family and plays an important role in cell division [13]. Survivin inhibits apoptosis by interaction with caspase-3 and -7. Another mechanism of apoptosis inhibition is related to the blocking of mitochondrial release of Smac/DIABLO complex and direct binding to IAP3 (inhibitor of apoptosis protein 3).

Survivin is involved in cancerogenesis and its enhanced expression has been found in many cancer 
types [13-15]. It has been suggested to be associated with higher tumor stage and cancer aggressiveness. Post-transcriptional phosphorylation is responsible for alternative survivin transcripts ratios, which may influence the regulatory balance between cytoprotection and proapoptotic action. The fulllength transcript coexists with four splice isoforms: DEx3, 3B, 2B, and 2A which have different biology. In general, survivin DEx3 and $3 \mathrm{~B}$ are considered anti-apoptotic, while $2 \mathrm{~B}$ and $2 \mathrm{~A}$ have pro-apoptotic properties. Moreover, survivin transcripts ratios might predict the response to chemotherapy [13].

Survivin can be expressed in healthy tissues with high proliferation potential, but the level of expression is much lower than in cancer cells. Survivin has been also detected in premalignant lesions, such as breast adenomas and colon polyps. Importantly, in precancerous tumors survivin was found mainly in the cytoplasm, while in cancers it dominated in the nuclear compartments [13].

Survivin has been investigated as a potential molecular biomarker of pituitary adenomas, but the obtained results are conflicting [16-20]. By using RT-qPCR method [21], in contrast to malignant endocrine tumors, we did not find over-expression of survivin and its splicing variants in pituitary adenomas, even if associated with local invasiveness. Therefore, the aim of this study was to evaluate survivin and its splicing variants survivin $2 \mathrm{~B}$ and $\mathrm{DEx} 3$ expression in pituitary adenomas at the protein level by the use of semi-quantitative immunohistochemistry.

\section{Material and methods}

Pituitary tissues. The study group consisted of eight tissue samples of pituitary adenomas: five of non-functional tumors, two of GH-secreting tumors, and one PRL-secreting tumor. Eight healthy pituitary tissue samples obtained from autopsy cases due to fatal trauma served as controls. Re- moved pituitary tissues were fixed in $10 \%$ formalin and embedded in paraffin wax. The study was approved by the local ethics committee, and informed written consent was obtained from each patient.

Immunohistochemical staining. Tissues were fixed in formalin, embedded in paraffin and $5-\mu \mathrm{m}$ sections were taken. Reactions were carried out with the use of rabbit monoclonal anti-survivin antibody (dilution 1:250, Abcam, ab76424), rabbit polyclonal anti-survivin DEx3 antibody (dilution 1:250, Abcam, ab3731), rabbit polyclonal anti-survivin 2B antibody (dilution 1:250, Abcam, ab3729). Reactions were visualized through subsequent incubations with a solution of biotinylated antibodies, streptavidin-horseradish peroxidase complex and diaminobenzidine (DAB) chromogen.

The intensity of survivin/survivin 2B/survivin DEx3 expressions was evaluated using the modified semi-quantitative immunoreactive score (IRS) approach, according to Remmele and Stegner [22], taking into account the percentage of positively stained cells (PP) (in the range of 0 to 4 points) and the staining intensity (SI) (in the range of 0 to 3 points). The total score ranges from 0 to 12 points $($ IRS $=\mathrm{SI} \times \mathrm{PP})$. Furthermore, the final score was presented on a five point scale ( 0 to 4 points) according to the formulas: 1-2 IRS points correspond to 1 point, 3-4 IRS points -2 points, $6-8$ IRS points -3 points, 9-12 IRS points -4 points.

Statistical analysis. Statistical analysis was performed with MedCalc version 15.8 (MedCalc Software bvba, Ostend, Belgium). P-value less than 0.05 indicated statistical significance. A comparison of the analyzed parameters between two groups was performed by the Mann-Whitney U test.

\section{Results}

Characteristics of the study group and the immunoreactivity of survivin and its splice variants $2 \mathrm{~B}$ and DEx3 expressions are provided in Table 1.

Table 1. Characteristics and survivin/survivin 2B/survivin DEx3 expressions in the study group

\begin{tabular}{|c|c|c|c|c|c|c|c|}
\hline \multirow{2}{*}{$\begin{array}{l}\text { Case } \\
\text { No. }\end{array}$} & \multirow{2}{*}{$\begin{array}{l}\text { Hormonal activity } \\
\text { of the tumor }\end{array}$} & \multirow{2}{*}{$\begin{array}{c}\text { Sex }(F-\text { female } \\
\quad M-\text { male })\end{array}$} & \multirow{2}{*}{$\begin{array}{c}\text { Age } \\
\text { (years) }\end{array}$} & \multirow{2}{*}{$\begin{array}{l}\text { Size } \\
{[\mathrm{mm}]}\end{array}$} & \multicolumn{3}{|c|}{ IHC staining intensity } \\
\hline & & & & & Survivin & Survivin 2B & Survivin DEx3 \\
\hline 1. & Non-functioning & M & 68 & 40 & 0 & 0 & 0 \\
\hline 2. & GH-secreting & $\mathrm{F}$ & 56 & 17 & 1 & 0 & 0 \\
\hline 3 & Non-functioning & M & 47 & 45 & 1 & 1 & 0 \\
\hline 4. & Non-functioning & $\mathrm{F}$ & 42 & 31 & 2 & 1 & 0 \\
\hline 5 & Non-functioning & $\mathrm{F}$ & 42 & 37 & 1 & 1 & 0 \\
\hline 6. & PRL-secreting & M & 57 & 20 & 4 & 0 & 2 \\
\hline 7. & GH-secreting & $\mathrm{F}$ & 45 & 21 & 4 & 3 & 2 \\
\hline 8. & Non-functioning & $\mathrm{F}$ & 48 & 33 & 2 & 3 & 0 \\
\hline
\end{tabular}



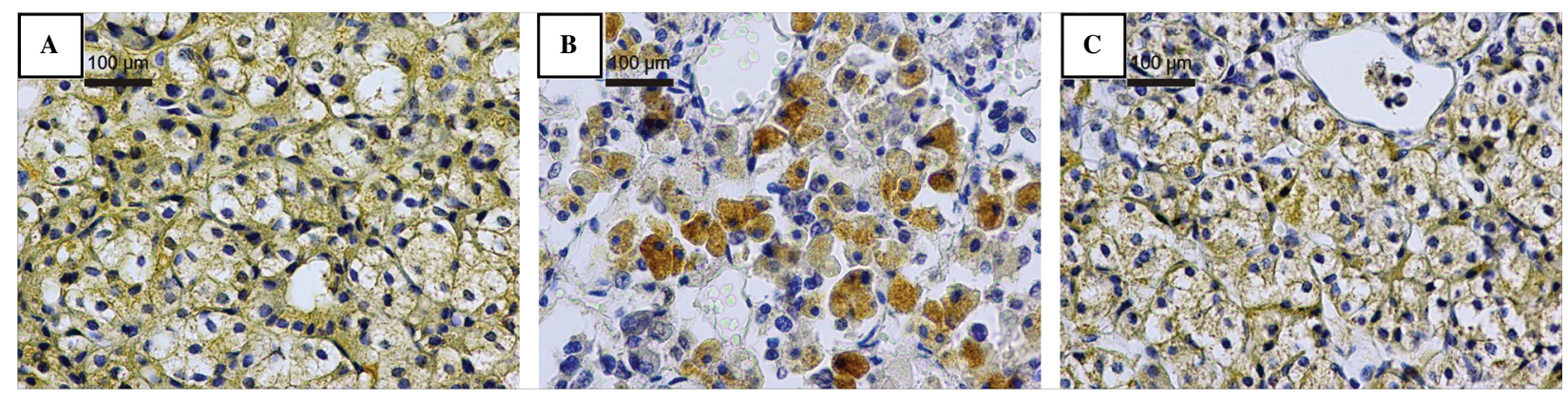

Figure 1. Cytoplasmatic localization of the expression of survivin (A), survivin DEx3 (B) and survivin 2B (C) in pituitary tumor. The studied proteins were visualized by immunohistochemistry as described in Material and methods. Scale bars: $100 \mu \mathrm{m}$.
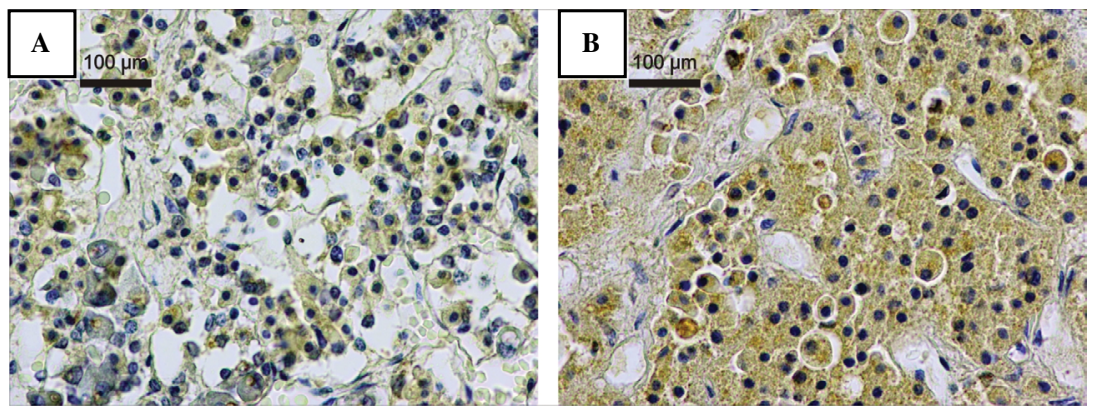

Figure 2. Comparative immunohistochemical staining for survivin $2 \mathrm{~B}$ in non-functioning pituitary adenoma (A) and normal pituitary gland (B). Scale bars: $100 \mu \mathrm{m}$.

The median age of patients with pituitary adenomas was 47.5 years (interquartile range (IQR) $43.5-56.5 \mathrm{yrs})$ and that of control subjects 48.0 years (IQR 44-56.5 yrs) $(\mathrm{p}=0.9547)$. Survivin expression was found in $87.5 \%$ of the study group and in $100 \%$ of the controls. A positive staining of survivin $2 \mathrm{~B}$ was found in $62.5 \%$ of pituitary adenomas and in $100 \%$ of controls. Survivin DEx 3 was recognized in $25 \%$ of pituitary adenomas and in $12.5 \%$ of normal pituitary glands (Table 1). Immunostaining of pituitary adenomas was not uniform. Survivin and its splice variants $\mathrm{DEx} 3$ and $2 \mathrm{~B}$ were predominantly localized in the cytoplasm of pituitary tumors and controls (Fig. 1).

Figure 2 shows staining of survivin $2 \mathrm{~B}$ in a pituitary adenoma and normal pituitary gland. The immunoreactivity of survivin $2 B(p=0.0498)$ was significantly lower in pituitary adenomas when compared with normal pituitary glands (Fig. 3).

\section{Discussion}

We aimed to investigate survivin and its splice variants DEx 3 and 2B expression in pituitary adenomas and normal pituitary glands using immunohistochemistry. We found down-regulation of survivin 2B expression in pituitary adenomas, while the intensity of survivin

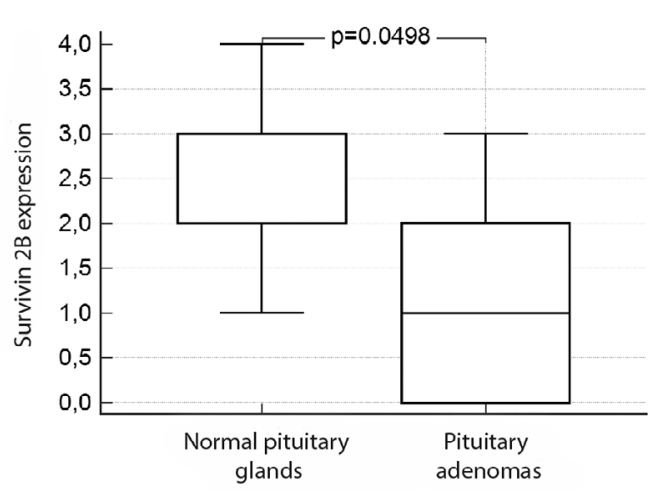

Figure 3. Comparison of survivin 2B expression in pituitary adenomas and normal pituitary glands. Central box represents the values from the lower to upper quartile $\left(25^{\text {th }}\right.$ to $75^{\text {th }}$ percentile). The middle line represents the median. Whiskers extend to a multiple of $1.5 \times$ the distance of the upper and lower quartile, respectively.

and survivin DEx3 immunoreactivity did not differ between pituitary tumors and normal glands. To the best of our knowledge, this is the first study evaluating immunohistochemical expression of survivin and its splice variants DEx3 and 2B in pituitary adenomas.

Survivin expression in pituitary tumors has been studied previously, but results are contradictory. We 
have previously found that the expression of survivin and its splice variants DEx3 and $2 \mathrm{~B}$ at the $\mathrm{m} R N A$ level in 50 pituitary adenomas was similar to that in 12 normal pituitary glands [21]. Formosa et al. analyzed immunohistochemical expression of survivin in pituitary adenomas and healthy pituitary tissues [19]. They did not find the expression of survivin in normal pituitary glands, whereas its expression in pituitary tumors was extremely low. Zhang et al. observed higher survivin expression in invasive pituitary adenomas, whereas other authors did not confirm these findings [17, 20, 21].

Increased cell proliferation and decreased apoptosis [5] were found in pituitary adenomas, so the latter might explain observed decreased survivin 2B expression. The regulation of cell proliferation in invasive pituitary adenomas is thought to be disturbed [5], although it has not been reflected in our study by potential up-regulation of anti-apoptotic proteins survivin and survivin DEx3. Survivin 2B down-regulation has been also observed in gastric cancers, with further decrease in late-stage tumors [23]. Moreover, decreased survivin $2 \mathrm{~B}$ expression had prognostic value in gastric cancers, which supports its potential role in tumor progression. In view of these observations, the down-regulation of survivin 2B in pituitary adenomas might also suggest its potential involvement in pituitary neoplastic transformation. Despite pituitary adenomas being benign lesions with slow progression, some of them are invasive and recurrent. Therefore, our results might have clinical implications indicating potential therapeutic targets. Interestingly, we have not found over-expression of survivin DEx3, which is considered a negative prognostic marker in many malignant tumors [13]. However, we observed cytoplasmic staining of survivin and its splice variants, which is consistent with other studies of benign lesions, in contrast to nuclear staining observed in malignant cells [13].

In summary, our preliminary data suggest that survivin and its splice variants might be involved to some extent in benign tumor growth of pituitary adenomas. However, survivin cannot be regarded as a candidate for targeted therapy or molecular biomarker of pituitary adenomas.

\section{Limitation of the study}

Due to the limited sample size we were not able to investigate a possible association between survivin/its splice variants DEx3 and 2B and hormonal activity of the pituitary adenomas.

\section{Conflict of interest}

The authors declare that they have no conflict of interest.

\section{Acknowledgments}

The study was supported by the of The Polish Committee for Scientific Research Award, grant No. N N402 523640.

\section{References}

1. Ezzat S, Asa SL, Couldwell WT, et al. The prevalence of pituitary adenomas: a systematic review. Cancer. 2004; 101(3): 613-619, doi: 10.1002/cncr.20412, indexed in Pubmed: 15274075 .

2. Fernandez A, Karavitaki N, Wass JAH. Prevalence of pituitary adenomas: a community-based, cross-sectional study in Banbury (Oxfordshire, UK). Clin Endocrinol (Oxf). 2010; 72(3): 377-382, doi: 10.1111/j.1365-2265.2009.03667.x, indexed in Pubmed: 19650784.

3. Gruppetta M, Mercieca C, Vassallo J. Prevalence and incidence of pituitary adenomas: a population based study in Malta. Pituitary. 2013; 16(4): 545-553, doi: 10.1007/s11102012-0454-0, indexed in Pubmed: 23239049.

4. Vandeva S, Tichomirowa MA, Zacharieva S, et al. Genetic factors in the development of pituitary adenomas. Endocr Dev. 2010; 17: 121-133, doi: 10.1159/000262534, indexed in Pubmed: 19955762.

5. Jiang $\mathrm{X}$, Zhang $\mathrm{X}$. The molecular pathogenesis of pituitary adenomas: an update. Endocrinol Metab (Seoul). 2013; 28(4): 245-254, doi: 10.3803/EnM.2013.28.4.245, indexed in Pubmed: 24396688.

6. Dworakowska D, Grossman AB. The molecular pathogenesis of pituitary tumors: implications for clinical management. Minerva Endocrinol. 2012; 37: 157-172, indexed in Pubmed: 22691889

7. Jia W, Lu R, Jia G, et al. Expression of pituitary tumor transforming gene (PTTG) in human pituitary macroadenomas. Tumour Biol. 2013; 34(3): 1559-1567, doi: 10.1007/s13277-0130686-2, indexed in Pubmed: 23404407.

8. Li Y, Zhou LP, Ma P, et al. Relationship of PTTG expression with tumor invasiveness and microvessel density of pituitary adenomas: a meta-analysis. Genet Test Mol Biomarkers. 2014; 18(4): 279-285, doi: 10.1089/gtmb.2013.0447, indexed in Pubmed: 24611443.

9. Fukuoka H, Takahashi Y. The role of genetic and epigenetic changes in pituitary tumorigenesis. Neurol Med Chir (Tokyo). 2014; 54 Suppl 3(12): 943-957, doi: 10.2176/nmc.suppl.2014-3, indexed in Pubmed: 25877147.

10. Seltzer J, Ashton CE, Scotton TC, et al. Gene and protein expression in pituitary corticotroph adenomas: a systematic review of the literature. Neurosurg Focus. 2015; 38(2): E17, doi: 10.3171/2014.10.FOCUS14683, indexed in Pubmed: 25639319.

11. Pawlikowski M, Radek M, Kunert-Radek J, et al. Overexpression of prothymosin alpha is related to pituitary adenoma recurrence but not to adenoma invasiveness and proliferation. Endokrynol Pol. 2014; 65(5): 382-386, doi: 10.5603/EP.2014.0053, indexed in Pubmed: 25301489. 
12. Żebracka-Gala J, Rudnik A, Hasse-Lazar K, et al. Molecular classification of pituitary adenomas: in search for criteria useful for high-throughput studies. Endokrynol Pol. 2016; 67(2): 148-156, doi: 10.5603/EP.a2016.0024, indexed in Pubmed: 26884293.

13. Waligórska-Stachura J, Jankowska A, Waśko R, et al. Survivin - prognostic tumor biomarker in human neoplasms - review. Ginekol Pol. 2012; 83(7): 537-540, indexed in Pubmed: 22880480.

14. Sawicka-Gutaj N, Waligórska-Stachura J, Andrusiewicz M, et al. Nicotinamide phosphorybosiltransferase overexpression in thyroid malignancies and its correlation with tumor stage and with survivin/survivin DEx3 expression. Tumour Biol. 2015; 36(10): 7859-7863, doi: 10.1007/s13277-015-3506-z, indexed in Pubmed: 25946974.

15. Waligórska-Stachura J, Andrusiewicz M, Sawicka-Gutaj N, et al. Survivin delta Ex3 overexpression in thyroid malignancies. PLoS One. 2014; 9(6): e100534, doi: 10.1371/journal. pone.0100534, indexed in Pubmed: 24945990.

16. Wasko R, Waligorska-Stachura J, Jankowska A, et al. Coexpression of survivin and PCNA in pituitary tumors and normal pituitary. Neuro Endocrinol Lett. 2009; 30(4): 477-481, indexed in Pubmed: 20010504.

17. Jankowska A, Wasko R, Waligorska-Stachura J, et al. Survivin products in pituitary tumors. Neuro Endocrinol Lett. 2008; 29(6): 1033-1037, indexed in Pubmed: 19112393.
18. Wasko R, Jankowska A, Waligorska-Stachura J, et al. Survivin expression in pituitary adenomas. Neuro Endocrinol Lett. 2005; 26(3): 209-212, indexed in Pubmed: 15990723.

19. Formosa R, Gruppetta M, Falzon S, et al. Expression and clinical significance of Wnt players and survivin in pituitary tumours. Endocr Pathol. 2012; 23(2): 123-131, doi: 10.1007/s12022-012-9197-8, indexed in Pubmed: 22327960.

20. Zhang Yc, Gao J, Xin T, et al. Expression of survivin in invasive pituitary adenoma. Saudi Med J. 2008; 29(11): 1589-1592, indexed in Pubmed: 18998006.

21. Waligórska-Stachura J, Andrusiewicz M, Sawicka-Gutaj N, et al. Evaluation of survivin splice variants in pituitary tumors. Pituitary. 2015; 18(3): 410-416, doi: 10.1007/s11102-014-0590-9, indexed in Pubmed: 25107550.

22. Remmele W, Stegner HE. Vorschlag zur einheitlichen Definition eines immunreaktiven Score (IRS) fur den Immunohistochemichen Ostrogenrezeptor-Nachweis (ER-ICA) im Mammikarzinomgewebe. Patologie. 1987; 8: $138-140$.

23. Krieg A, Mahotka C, Krieg T, et al. Expression of different survivin variants in gastric carcinomas: first clues to a role of survivin-2B in tumour progression. Br J Cancer. 2002; 86(5): 737-743, doi: 10.1038/sj.bjc.6600153, indexed in Pubmed: 11875736 .

Submitted: 19 August, 2016

Accepted after reviews: 17 March, 2017 Available as AoP: 23 March, 2017 